\title{
Factors affecting public dissatisfaction with urban family physician plan: A general population based study in Fars Province
}

\author{
Mohammad Hadi Imanieh ${ }^{1}$, Alireza Mirahmadizadeh ${ }^{2}$, Bahareh Imani ${ }^{3}$
}

${ }^{1}$ M.D. Professor of Pediatrics, Department of Pediatrics, Shiraz University of Medical Sciences, Shiraz, Iran

${ }^{2}$ M.D. MPH, Ph.D. of Epidemiology, Non-Communicable Diseases Research Center, Shiraz University of Medical Sciences, Shiraz, Iran

${ }^{3}$ M.D. Shiraz University of Medical Sciences, Shiraz, Iran

Type of article: Original

\begin{abstract}
Background: Understanding the level of public satisfaction with a family physician plan as well as the relevant factors in this respect, can be employed as valuable tools in identifying quality of services.

Objective: To determine the factors affecting public dissatisfaction with an urban family physician plan in Iran. Methods: This cross-sectional study was conducted from January 2014 through June 2015 on Fars Province residents in Iran, selected based on cluster sampling method. The data collection instrument was comprised of a two-part checklist including demographic information and items related to dissatisfaction with the family physician plan, specialists, para-clinic services, pharmacy, physicians on shift work, emergency services, and family physician assistants. Data were described by SPSS 20.

Results: In this study, 1,020 individuals (524 males, 496 females) were investigated. Based on the results, the most frequent factor affecting dissatisfaction with physicians was their single work shifts and unavailability (53\%). In terms of dissatisfaction with family physicians' specialist colleagues and para-clinic services, the most common factors were related to difficulty in obtaining a referral form (41.5\%) and making appointments $(21.6 \%)$, respectively. Given the level of dissatisfaction with pharmacies, the significant factor was reported to be excessive delay in medication delivery $(31.6 \%)$; and in terms of physicians on shift work and emergency services, the most important factor was lower work hours for family physicians $(9.2 \%)$.

Conclusion: It seems that, the most common causes of dissatisfaction with the urban family physician plan are due to the short duration of services, obtaining a referral form and making appointments, and providing prescribed medications.
\end{abstract}

Keywords: Satisfaction, Family Physician, Referral System

\section{Introduction}

Without healthcare, many opportunities to improve basic health are lost (1). Low-cost and easy access to healthcare centers for patients is taken into account as the significant factors in developed societies. In this respect, all countries make attempts to achieve this privileged status and a family physician plan is one of the steps in this regard (2). Moreover, the World Health Organization recognizes the family physician as the center of global efforts to improve quality, cost-effectiveness, and equity in health care systems (1-2). Accordingly; equity, quality, efficiency, development of a referral system, increased accountability in the health market, reduction in unnecessary costs, more access to health services by the poor as well as higher coverage of health services are among the objectives of the health care system and the family physician plan (3). In addition, patient satisfaction is one of the measures indicating high efficiency of health service providers (2). Therefore, research on patient expectations of physicians allows for a fundamental approach to understanding the relationship between physicians and patients that leads to the consideration of patient expectations, and not to make decisions absolutely tied to physicians' judgments $(4,5)$.

\section{Corresponding author:}

Assistant Professor Dr. Alireza Mirahmadizadeh, Department of Epidemiology, Non-communicable Research Center. Shiraz University of Medical Sciences, Shiraz, Iran.

Tel: +98.7132122320, Fax:+98.7132347315, Mobile: +98.9125169146, Email: mirahamdia@sums.ac.ir Received: November 12, 2016, Accepted: June 16, 2017, Published: November 2017 iThenticate screening: June 17, 2017, English editing: August 02, 2017, Quality control: August 16, 2017 (C) 2017 The Authors. This is an open access article under the terms of the Creative Commons Attribution-NonCommercialNoDerivs License, which permits use and distribution in any medium, provided the original work is properly cited, the use is non-commercial and no modifications or adaptations are made. 
It seems there is a need to make major changes in the delivery of healthcare services in societies, together with global developments in medical education to satisfy most patients, which has been confirmed in several countries through numerous research studies (6). In this respect, patient satisfaction as an important index to assess the quality and delivery of services, is dependent on medical staff and employees (7). One of the main objectives of Iran's Fifth Development Plan in terms of economic, cultural and social aspects in the field of health, is the implementation of a family physician plan and referral system (4-6). It seems that the commencement of such a great and comprehensive plan in Iran is in need of monitoring the results of such a plan properly (3-5). Previous studies have shed light on the factors affecting dissatisfaction of rural individuals with the family physician plan, including unavailability of physicians, non-residence of physicians in rural areas due to being non-native, and movement difficulties of physicians from urban areas to rural ones which could originate from low tariffs for rural insurance, referrals at special hours, as well as downsized human forces and inappropriate time management in healthcare centers (8). Studies conducted on family physicians have also revealed that $30 \%$ of Fars Province residents were dissatisfied with the urban family physician plan (9). However, factors associated with this discontent have not been examined in a comprehensive study. Provided that an awareness of the causes of public dissatisfaction with the services of family physicians can be obtained, the family physician plan will be promoted and the public can be more satisfied through identifying problems and weaknesses in such a plan as well as making changes in health planning. Therefore, the aim of this study was to determine the factors affecting dissatisfaction of Fars Province residents with the services of family physicians affiliated by Shiraz University of Medical Sciences.

\section{Material and Methods}

\subsection{Study design and setting}

This cross-sectional study was conducted from January 2014 through June 2015 on Fars Province residents in Iran.

\subsection{Inclusion and Exclusion Criteria}

To begin with, participants completed a questionnaire of which validity and reliability had been approved in an earlier study (9). Individuals who scored lower than 2.5 points on a Likert scale in mentioned questionnaires were assumed equivalent to being dissatisfied with the family physician plan, and were deemed eligible to take part in the study. Membership of participants in the family physician plan aged over 18 years, of Iranian nationality, have residence in Fars Province, and have insurance coverage were also among other inclusion criteria in the present study. Furthermore, patients unable to respond to the questionnaire, or those unwilling and discontent to participate in the study were excluded.

\subsection{Sample Size}

Given the $95 \%$ confidence level for the causes of dissatisfaction and also volatility of each of the causes, $50 \%$ ratio to estimate the maximum sample size and $10 \%$ relative error (estimation error of $5 \%$ ) were considered to achieve the required sample size. Using the cluster sampling method in this study, variance inflation factor or design effect was considered approximately 2.6 in order to increase the accuracy of sampling, and a total sample size of 1,000 individuals was obtained.

\subsection{Instrument and data collection}

The data collection instrument was comprised of a two-part checklist including demographic information and items related to dissatisfaction with family the physician plan, specialists, para-clinic services, pharmacy, physicians on shift work, emergency services, and family physician assistants. The items of dissatisfaction included unavailability of family physicians, long waiting times to receive services, lack of a suitable environment for the delivery of services, inappropriate conduct by the family physician team, absence of patient education for prevention of diseases, inadequate visit time for patients, dissatisfaction with the academic competence of family physicians, discontent with the referral system, and dissatisfaction with costs.

\subsection{Statistical Analysis}

All the data were analyzed through the IBM SPSS Statistics for Windows (IBM Corp, version 20, Armonk, NY) and descriptive statistics including absolute and relative frequencies were used to show the frequency of factors affecting dissatisfaction.

\subsection{Ethical Considerations}

This study was approved by the Ethics Committee of Shiraz University of Medical Sciences. The participants could be excluded at any stage and there was no compulsion for people to participate in the study (8947-77-01-93). 
http://www.ephysician.ir

Moreover, this study was conducted in an observational form and no intervening attempts were made on participants.

\section{Results}

The study was conducted on 1,020 participants (524 men, 496 women). The majority of the study participants were married (74.0\%) with higher level or diploma equivalent education (60.8\%). Among the participants, 51.9\% had Iranian Health Insurance, $47 \%$ had Social Security Insurance while 1.2\% of participants were also covered by Relief Committee Insurance and in general $23.8 \%$ had Supplementary Insurance coverage. The results of this study showed that the most common causes of dissatisfaction were the unavailability of family physicians (53\%) and difficulty in making appointments (51\%); while the most frequent causes of dissatisfaction with family physician's specialist colleagues were the difficulty in obtaining the referral form (41.5\%) and making appointments and long waiting times to see a physician (29\%) (Table 1). Moreover, the most significant cause of dissatisfaction with family physician assistants (midwives, nurses, and technicians in public health or family health) was the long waiting times to receive services $(13.8 \%)$ (Table 2$)$. The most frequent causes of dissatisfaction with physicians on shift work and emergency services were low shift times (9.2\%) and lack of uniformity in payments (7.8\%) at various centers (Table 2). The greatest cause of dissatisfaction with para-clinic services (such as physiotherapy, laboratory, radiology, electrocardiogram, etc.) were associated with difficulty in making appointments and high expectations (21.6\%) and requests for referral forms $(13.6 \%)$, respectively (Table 2). Furthermore, the most common cause of dissatisfaction with pharmacies was related to delays and long waiting times to receive medications $(31.6 \%)$ as well as high costs $(25.8 \%)$ (Table 2$)$.

Table 1. Distribution of factors related to dissatisfaction with general practitioners and specialists of the Family Physician Plan in Fars Province in 2014-2015

\begin{tabular}{|c|c|c|c|}
\hline $\begin{array}{l}\text { Practitioners and } \\
\text { services }\end{array}$ & Factors & $\mathrm{n}$ & $\%$ \\
\hline \multirow[t]{10}{*}{ General practitioners } & High costs of visit & 26 & 2.5 \\
\hline & Unfavorable behavior of physician & 31 & 3.0 \\
\hline & Insufficient time for examinations and communication & 79 & 7.7 \\
\hline & High costs of injection services & 97 & 9.5 \\
\hline & Inadequate duration of educational and prevention notifications & 115 & 11.2 \\
\hline & Misdiagnosis or mistreatment & 122 & 11.9 \\
\hline & Inappropriate environment of physicians' offices & 133 & 13.0 \\
\hline & Unwillingness of physicians to issue referral forms & 153 & 15.0 \\
\hline & Difficulty in making appointments and long waiting times to see a physician & 520 & 51.0 \\
\hline & Office time limit (single work shifts, off weekends) & 541 & 53.0 \\
\hline \multirow[t]{10}{*}{ Specialists } & Insufficient time for examinations and communication & 14 & 1.3 \\
\hline & Unfavorable behavior of physician & 20 & 1.9 \\
\hline & Inadequate duration of educational and prevention notifications & 53 & 5.2 \\
\hline & High costs of visit & 89 & 8.7 \\
\hline & Inappropriate environment of physicians' offices & 90 & 8.8 \\
\hline & Unwillingness of physicians to visit the patients without referral forms & 90 & 8.8 \\
\hline & No cooperation with family physicians & 170 & 16.5 \\
\hline & $\begin{array}{l}\text { Unwillingness of physicians to revisit the patients without referral forms for } \\
\text { their follow-up procedures }\end{array}$ & 190 & 18.4 \\
\hline & Difficulty in making appointments and long waiting times to see a physician & 296 & 29.0 \\
\hline & Unwillingness of physicians to issue referral forms & 424 & 41.5 \\
\hline \multirow{7}{*}{$\begin{array}{l}\text { Night shifts / } \\
\text { Emergency services }\end{array}$} & High costs of services & 34 & 3.3 \\
\hline & No patients' admission & 45 & 4.4 \\
\hline & Difficulty in having access to medical services & 52 & 5.1 \\
\hline & Difficulty in making appointments and long waiting times to see a physician & 78 & 7.6 \\
\hline & Inappropriate environment of physicians' offices & 79 & 7.7 \\
\hline & Lack of uniformity in the amount of payments in different 24 -hour centers & 80 & 7.8 \\
\hline & Low work shift times of family physicians & 94 & 9.2 \\
\hline
\end{tabular}


Table 2. Distribution of factors related to dissatisfaction with physicians' assistants, para-clinic and pharmacies services of Family Physician Plan in Fars Province in 2014-2015

\begin{tabular}{|c|c|c|c|}
\hline $\begin{array}{l}\text { Practitioners and } \\
\text { services }\end{array}$ & Factors & $\mathrm{n}$ & $\%$ \\
\hline \multirow[t]{4}{*}{ Physicians' Assistants } & Unfavorable behavior of Assistants & 38 & 3.7 \\
\hline & High costs of visit & 52 & 5.1 \\
\hline & Unfavorable quality of procedures done by assistants & 77 & 7.5 \\
\hline & Long waiting times of receiving services & 141 & 13.8 \\
\hline \multirow{7}{*}{$\begin{array}{l}\text { Para-clinic services } \\
\text { (such as physiotherapy, } \\
\text { laboratory, radiology, } \\
\text { ECG, etc.) }\end{array}$} & Unfavorable behavior of service providers & 36 & 3.5 \\
\hline & Low quality of devices provided for para-clinic services & 62 & 6.0 \\
\hline & No cooperation with family physicians & 83 & 8.1 \\
\hline & Inappropriate environment of the centers providing services & 108 & 10.5 \\
\hline & High costs of services & 115 & 11.2 \\
\hline & Requests for referral forms and obligations to obtain them & 140 & 13.6 \\
\hline & Difficulty in making appointments and long waiting times to see a physician & 221 & 21.6 \\
\hline \multirow[t]{5}{*}{ Pharmacies } & No cooperation with family physicians & 83 & 8.10 \\
\hline & Inadequate instructions on how to take medications & 118 & 11.50 \\
\hline & Lack of medications & 186 & 18.2 \\
\hline & High costs despite being free, up to $300,000-500,000$ Rials & 264 & 25.80 \\
\hline & Excessive delay in medication delivery & 323 & 31.60 \\
\hline
\end{tabular}

\section{Discussion}

Nearly eight years after the onset of the rural family physician plan and its achievements in terms of increased community health and public satisfaction, authorities and policy-makers decided to expand such a plan in the urban system. Therefore, the urban family physician plan was implemented in the provinces of Fars and Mazandaran in 2012. After 2.5 years of implementation of the urban family physician plan, the level of satisfaction in participants was measured in a research study and the results showed that the level of satisfaction with the plan in $15.8 \%$ of participants was high, $31.3 \%$ of individuals had an average level of satisfaction, and $31.1 \%$ had low satisfaction in this respect (9). With regard to the rights of individuals in the family physician system and their expectations of the plan, a questionnaire was designed in collaboration with policy-makers and directors of the family physician plan, in order to identify the causes of dissatisfaction in the group with a low level of satisfaction (31.1\%). In the present study, seven items including family physician competence, time for examination and listening to patient's descriptions, access to family physician if required, complexity of referral system, waiting times to receive services, rate of referrals to family physicians, ways to deal with clients in different sectors of the family physician plan (family physicians, specialists, para-clinic services, pharmacy, physicians on work shift and emergency services, colleagues and assistants of family physicians) were investigated. The results of this study indicated that the most frequent cause of dissatisfaction with family physicians was associated with single work shifts and unavailability of physicians particularly on weekends, and visit time limits after 8 p.m. (53\%) which was higher than such dissatisfaction level with rural family physicians. The second cause of dissatisfaction was identified as long waiting times to receive services (51\%); while, in Slovenia, access to physician services and waiting times for services were recognized as the most and the least frequent factors affecting satisfaction with the family physician plan, respectively. Moreover, $51.6 \%$ and $49.1 \%$ of Slovenian respondents expressed their satisfaction with counseling time and possibility of raising patient problems with family physicians, respectively. However, in the present study, only $7.7 \%$ of participants were dissatisfied with inappropriateness of the method adopted and the time spent to listen to patients' descriptions. Such findings revealed the strength of physicians in this field that was consistent with the results of studies conducted in the US as well as those in the rural family physician plan $(10,11)$. Difficulty in obtaining a referral form was taken into account as the most common cause of dissatisfaction (41.5\%) with specialists, which could reflect weaknesses in culture-building in terms of the referral system among individuals. It should be noted that a very efficient referral system is also dominating in developed countries (12-13) and there are also concerns about lack of control over bureaucracy and access to specialists (14), but this issue has not led to any public dissatisfaction. However, insistence and urges by patients for some unnecessary services has become a challenge in the rural family physician plan. The results of previous studies showed that $56 \%$ of the referrals were not based on physicians' willingness but urges made by patients $(15,16)$. This demonstrated that trust and belief in the performance of family physicians was low in society and further investigations have also confirmed this (11). According to research studies, communication skills training for physicians could have a significant impact on 
patient satisfaction in this respect (17). It should be noted that family physicians are taken into account as the most influential individuals in familiarizing people with the family physician, and studies into the rural family physician plan show that physicians' share in terms of giving information to the public in this respect is low despite their significant roles (18). Therefore, absence of a referral system culture among people, inadequate and outdated scientific level of general practitioners, and spending less time and having no patience to give awareness about family physician laws and regulations in order to justify households covered by the family physician plan, were included as the causes of dissatisfaction with the referral system (18.6\%). Study participants also expressed their dissatisfaction with repeated requests for referral forms to read the test results. One of the contributing factors to discontent in this regard, was the public's unawareness of the laws and regulations associated with the family physician plan because the referral forms were not required up to a week after visits with specialists and lack of giving awareness to patients by specialists in this regard caused dissatisfaction among them. Of the participants in the study, 29\% were dissatisfied with long waiting times for visits with specialists; however, as the plan progressed and the referrals reduced, such dissatisfaction also decreased, which was in agreement with the results of other studies $(12,13,16)$.

Obtaining the referral form was considered as one of the causes of dissatisfaction with para-clinic services. It should be noted that there was no need to acquire referral forms for para-clinic services and the related procedure could be done with the same insurance. Thus, requests for referral forms to provide para-clinic services were illegal, and individuals were required to get informed in this respect. It should be noted that all the factors affecting satisfaction were taken into consideration in this study and in terms of dissatisfaction with pharmacies; excessive delay in the delivery of medications was not related to the family physician plan although it was among expectations and demands by the public in other provinces in Iran. Given the high level of costs for medications and despite being declared free-of-charge, it should be noted that the medications covered by the insurance organization could be free provided that they were prescribed by family physicians and the patients only had to pay the tariffs for technical costs of pharmacies as well as costs for free, strengthening, or foreign medications. Also, for medicines prescribed by a specialist, the patient was only obliged to pay $30 \%$ of the total amount as the added technical costs to the pharmacists. Therefore; the costs spent by Fars Province residents, if referred by family physicians, were much less than those in other provinces and indeed, they were associated with the expectations of individuals to get all medications completely free-of-charge, including free strengthening, and foreign medication. In terms of examination of causes for dissatisfaction with physicians on work shifts and emergency services, lack of uniformity in the amount of payments in 24-hour centers was highlighted. Thus, there was a need to have serious monitoring in this regard because the amount of payments should be the same and its opposite was considered as an offence. Periodical evaluation of each plan, in order to identify its strengths and weaknesses and to make revisions, is inevitable. Accordingly; developing family physician centers providing round-the-clock services, increasing the number of family physicians, and administering the centers through work shifts, can lower public dissatisfaction with single work shifts and unavailability of family physicians. Furthermore, cooperation among family physicians in terms of issuing referral forms based on instructions, and raising the scientific level of family physicians through "continuous education seminars", can increase public trust in family physicians and reduce urges for referral forms. In addition, taking courses in terms of family physician and the referral system in the internship period for medical students, can reduce medical errors and lead to increased public confidence in family physicians. Also, specialists can request revisits to family physicians through electronic medical records, and there is no need to obtain referral forms. In total, giving comprehensive information to the public, physicians, paramedics, and authorities in terms of laws and regulations, updated scientific information by family physicians, increased patience and time spent by family physicians, more monitoring and notification to unaware individuals and those who treat the implementation of some provisions with less attention in spite of knowledge of laws and regulations, were the main points emphasized in increasing the level of public satisfaction with the family physician plan. One of the limitations of this study was the high number of questions in the questionnaire, which, in some cases, lead to dissatisfaction of the participants.

\section{Conclusions}

It seems that, the factors related to dissatisfaction in each section of the family physician plan are different. As for general practitioners, its most common related factor is the domain of availability including, single work shifts, weekends off, and unavailability. However, for the specialists, unwillingness of physicians to issue referral forms was assumed as the main factor of dissatisfaction. Long waiting times of receiving services by physicians' assistants and delay in medication delivery by pharmacies were the other prominent factors related to dissatisfaction. 


\section{Acknowledgments:}

This paper was derived from a thesis completed by Dr. Bahareh Imani as a Medical Student (research proposal no. 87-1015). The study approved and financed by Vice-Chancellor for Research Affairs of Shiraz University of Medical Sciences. We also appreciate Dr. Karam Tavani, Mrs. Maryam Siadati, and Dr. Abdolrasul Hemmati for their cooperation in data collection.

\section{Conflict of Interest:}

There is no conflict of interest to be declared.

\section{Authors' contributions:}

All authors contributed to this project and article equally. All authors read and approved the final manuscript.

\section{References:}

1) Marmot M, Friel S, Bell R, Houweling TA, Taylor S. Commission on Social Determinants of Health. Closing the gap in a generation: health equity through action on the social determinants of health. Lancet. 2008; 372(9650): 1661-9. doi: 10.1016/S0140-6736(08)61690-6. PMID: 18994664.

2) Baker R. Development of a questionnaire to assess patients' satisfaction with consultations in general practice. Br J Gen Pract. 1990; 40(341): 487-90. PMID: 2282225, PMCID: PMC1371443.

3) Bagheri-lankarani K, Kalate Sadati A, Emanieh MH, Joolaei H, Moghadami M, Asemani O. A Review of Family Physician in Iran. Shiraz: Kosar; 2015.

4) Virji A, Britten N. A study of the relationship between patients attitudes and doctors prescribing. Fam Pract. 1991; 8(4): 314-9.

5) Armstrong D. What do patients want? BMJ. 1991; 303(6797): 261-2. doi: 10.1136/bmj.303.6797.261.

6) Yost NP, Bloom SL, Sibley MK, Lo JY, McIntire DD, Leveno KJ. A hospital-sponsored quality improvement study of pain management after cesarean delivery. Am J Obstet Gynecol. 2004; 190(5): 1341 6. doi: 10.1016/j.ajog.2003.10.707. PMID: 15167840 .

7) Williams B. Patient satisfaction: a valid concept? Soc Sci Med. 1994; 38(4): 509-16. doi: 10.1016/02779536(94)90247-X. PMID: 8184314.

8) Azizi J, Karimyar Jahromi M, Hojat M. Assessment of Darab County Villagers' Satisfaction with Family Doctor Functions from Different Aspects. J Fasa Univ Med Sci. 2012; 2(3): 193-8.

9) Honarvar B, lankarani KB, Ghaharmani S, Akbari M, Tabrizi R, Bagheri Z. Satisfaction and dissat is faction to ward urban family physician program: A population based study in shiraz, southern Iran. Int J Prev Med. 2016; 7(3). doi: 10.4103/2008-7802.173793. PMID: 26941904, PMCID: PMC4755284.

10) Jannati A, Maleki M, Gholizade M, Narimani M, Vakili S. Assessing the strengths and weaknesses of family physician program. knowledge \& health. 2010; 4(4): 38-43.

11) Ghorbani A, Nakhaie M, Dovlat Abadi A, Alemi H, Tabarraie Y. Study of satisfaction Rate of family Medicin Plan in insured population of sabzevar university Medical of sciences. Journal of sabzevar university of Medical science. 2013; 19(4): 364-70.

12) Karimi I, Salarian A, Zohreh A. A comparative study on equity in access to health services in developed countries and designing a model for Iran. Arak Medical University Journal. 2010; 12(4): 92 - 104.

13) Tawfik KA, Alshehri AM, Abdul Aziz F, Khwaja MS. Patterns of referral from health centers to hospital in Riyadh region. Eastern Mediterranean Health Journal. 1997; 3(2): 236-53.

14) Manca DP, Varnhagen S, Brelt MP, Allanca GM, Szafran O, Ausford A, et al. Rewards and challenges of family practice: Web-based survey using the Delphi method. Can fam physician. 2007; 53(2): 278-86. PMID: 17872645, PMCID: PMC1949127.

15) Pour Shirvani N, Amiri A, Motlagh ME, Kabir MJ, Maleki MR, Shabestani Monfared A, et al. Evaluation of the Function of Referral System in Family Physician Program in Northern Provinces of Iran: 2008. JBUMS. 2010; 11(6): 46-52.

16) van Uden CJ, Winkens RA, Wesseling GJ, Crebolder HF, van Schayck CP. Use of out of hours services: a comparsion between two organizations. Emerg Med J. 2003; 20(2): 184-7. doi: 10.1136/emj.20.2.184. PMID: 12642541, PMCID: PMC1726038.

17) Managheb SE, Firouzi H, Jafarian J. The impact of communication skills training based on calgarycambridge guideline on knowledge, attitude and practice of family phisicians in Jahrom University of medical sciences 2007. Jahrom Medical Journal. 2008; 6(2): 74-84.

18) Miri MR, Shafiee F, Hydrania AR, A. K. Study on behavior interntion model (BIM) to the attitude of tribement towards family planning. Journal of Mazandran University of Medical Sciences. 2003; 12(37): 67-73. 